\title{
Endoscopic ultrasound-guided ethanol ablation of insulinoma
}

\author{
Ewa Zalewska ${ }^{(1}$, Przemysław Kłosowski', Michał Dubowik², Rafał Pęksa ${ }^{3}$, Krzysztof Sworczak' \\ ${ }^{1}$ Department of Endocrinology and Internal Medicine, Medical University of Gdansk, Gdansk, Poland \\ ${ }^{2}$ Department of Gastroenterology and Hepatology, Medical University of Gdansk, Gdansk, Poland \\ ${ }^{3}$ Department of Pathology, Medical University of Gdansk, Gdansk, Poland
}

Key words: insulinoma; ethanol ablation; hypoglycemia

A 72-year-old female patient with a four-month history of recurrent episodes of symptomatic hypoglycaemia was admitted to the Department of Endocrinology and Internal Medicine, Medical University of Gdansk (Poland) in November 2019. A month earlier, the patient was hospitalised in the Cardiology Department of St. Wojciech hospital due to non-ST segment elevation myocardial infarction, where a low blood glucose level of $2.4 \mathrm{mmol} / \mathrm{L}$ was recorded during one of the episodes of agitation, excessive sweating, and weakness. In the Department of Endocrinology and Internal Medicine, insulinoma was confirmed on the basis of clinical presentation and additional examinations. The starvation test was positive. The criteria for endogenous hyperinsulinaemic hypoglycaemia were met after a fasting time of $5 \mathrm{~h}$ with the glucose level of $1.8 \mathrm{mmol} / \mathrm{l}$. Diffusion-weighted magnetic resonance imaging revealed a 12-mm lesion on the anterior surface of the pancreas, which did not show restriction diffusion (Fig. 1A) and typical arterial enhancement (Fig. 2B). Endoscopic ultrasound (EUS) elastography showed lower elasticity of the tumour as compared to that of the surrounding pancreatic tissue (Fig. 2). EUS-guided fine-needle aspiration of the lesion was performed to collect material for cytological examination. The cellular smears revealed a scant to moderate amount of granular cytoplasm, which indicated neurosecretory capability (Fig. 3).

The standard treatment for a functional pancreatic neuroendocrine tumour is surgical resection $[1,2]$. In terms of heart failure with low ejection fraction, the perioperative risk was high because of recent myocardial infarction with the necessity to continue double antiplatelet therapy. Considering this limitation and the patient's severity of symptoms and benign char-
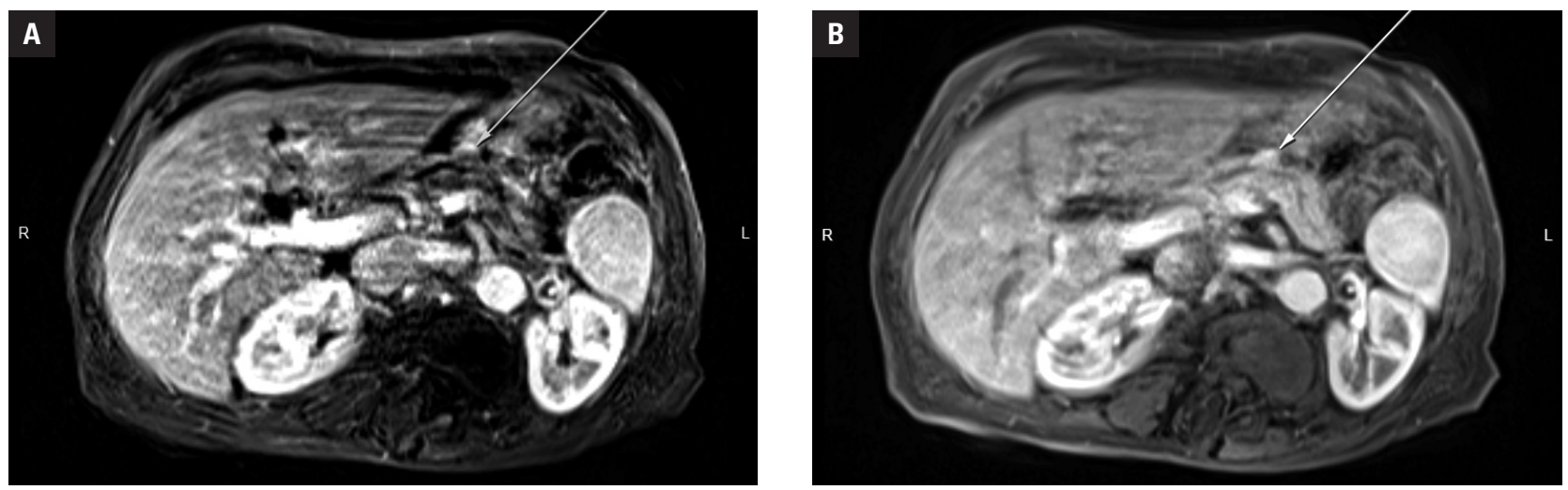

Figure 1. Magnetic resonance imaging of the abdomen revealed a 12-mm lesion on the anterior surface of the pancreas (arrow), which did not show restriction diffusion on diffusion-weighted imaging (A) and did not present typical contrast enhancement for insulinoma on T1-weighted images (B)

Ewa Zalewska, PhD, Department of Endocrinology and Internal Medicine, Medical University of Gdansk, ul. Debinki 7, 80-952 Gdańsk, Poland, tel: (+48) 5834928 40, fax: (+48) 5834928 41; e-mail: ewa.zalewska@gumed.edu.pl 


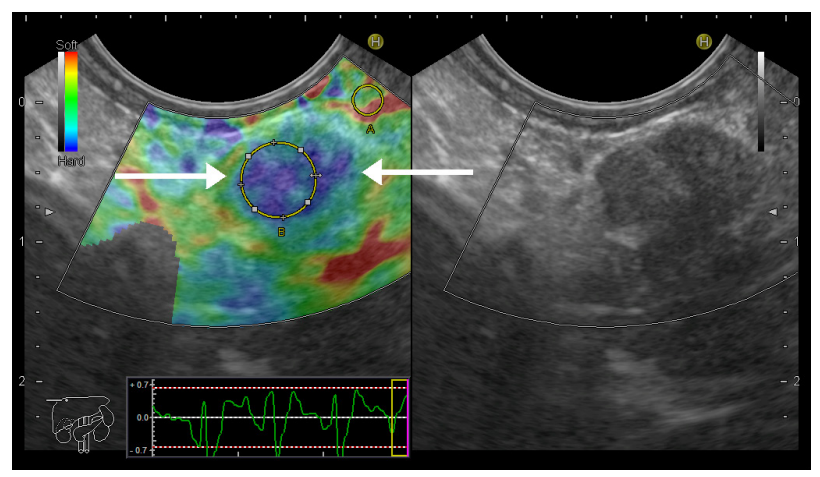

Figure 2. An endoscopic ultrasound elastography revealed lower elasticity of the tumour as compared to that of the surrounding pancreatic tissue (arrows)

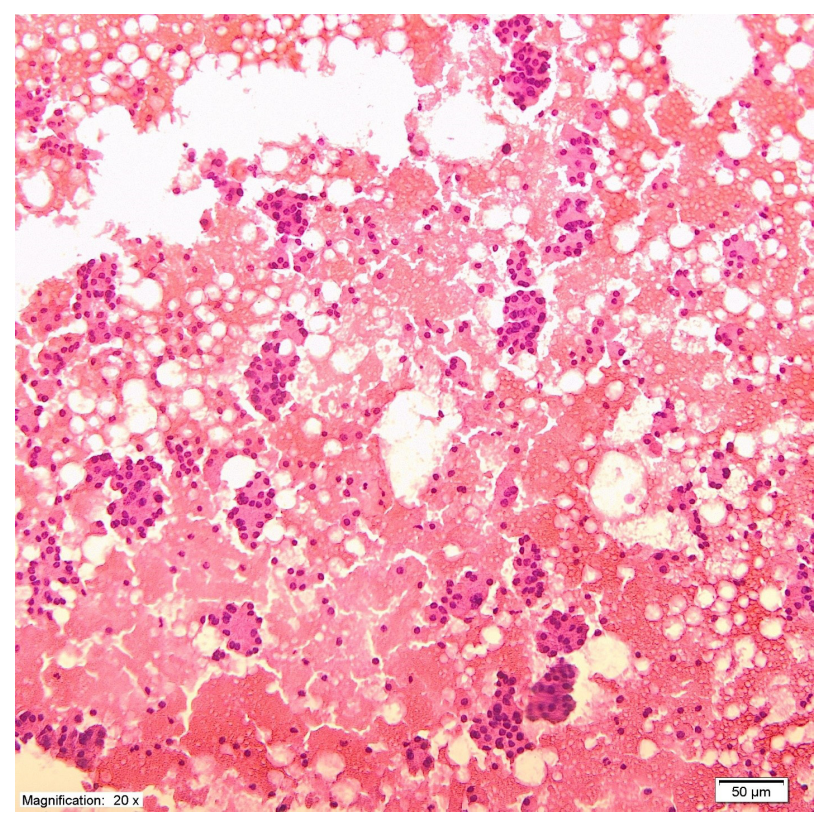

Figure 3. A smear from the pancreatic tumour showing a cellular aspirate; numerous isolated cells and sheets of tumour cells with overlapping and overcrowding of nuclei; minimal nuclear pleomorphism; fine, evenly dispersed nuclear chromatin with occasional inconspicuous nucleoli; a scant to moderate amount of granular, amphophilic cytoplasm (neurosecretory capability)

acteristic of insulinoma in most cases, we proposed less invasive treatment [1-5]. The patient underwent EUS-guided ethanol ablation of the lesion. Two millilitres of $96 \%$ ethanol were injected under visual control in small aliquots $(0.1 \mathrm{~mL})$ until hyperechoic blush was seen within the tumour (Fig. 4). After the procedure, a partial improvement was observed. The episodes of hypoglycaemia occurred less frequently, and the symptoms were milder. In January 2020, a second session of EUS-guided ethanol ablation was performed, and it seemed to be completely effective in a five-month follow-up. We did not observe any clinically significant adverse effects of the treatment approach. The

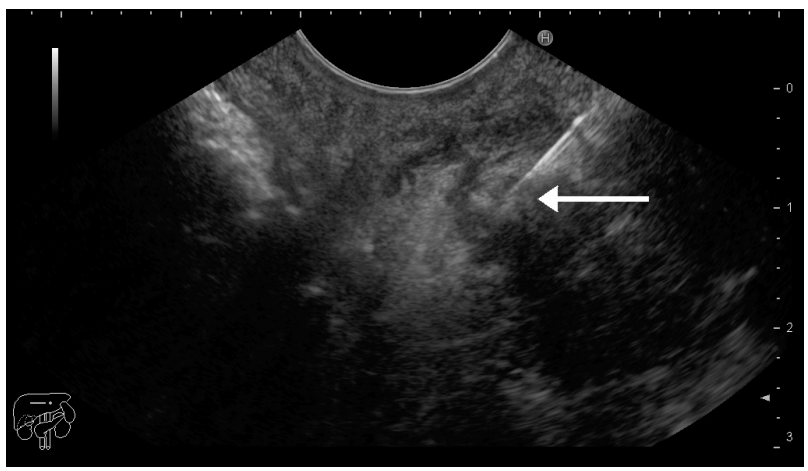

Figure 4. Endoscopic ultrasound-guided ethanol ablation of the insulinoma. Two millilitres of $96 \%$ ethanol were injected under visual control in small aliquots $(0.1 \mathrm{~mL})$ until hyperechoic blush was seen within the tumour (arrow)

patient reported mild abdominal pain on the day of the procedure, and a transient, six-fold elevation of lipase activity was observed, which was normalised within $72 \mathrm{~h}$. Another type of nonsurgical management of insulinoma is EUS-guided radiofrequency ablation. Both ablative therapies, namely ethanol injection and radiofrequency, seem to be effective, safe, and repeatable procedures in patients who are at increased surgical risk [1-5]. Mele et al. reported that after an average follow-up of 16 months, the overall success rate of nonsurgical ablative treatments of insulinoma was $84 \%$ [4]. The longest follow-up of a patient after a successful EUS-guided ethanol ablation of insulinoma was four years [5]. It is worth highlighting that ethanol ablation is more economically beneficial than EUS-guided radiofrequency ablation. Clinical trials and longer follow-ups are warranted to determine which of these nonsurgical ablative techniques achieves the best clinical outcome for the treatment of insulinoma [1-5].

\section{Conflict of interest}

None declared.

\section{References}

1. Buicko JL, Finnerty BM, Zhang T, et al. Insights into the biology and treatment strategies of pancreatic neuroendocrine tumors. Ann Pancreat Cancer. 2019; 2, doi: 10.21037/apc.2019.06.02, indexed in Pubmed: 31535089.

2. Kos-Kudła B, Blicharz-Dorniak J, Strzelczyk J, et al. Consensus Conference, olish Network of Neuroendocrine Tumours. Pancreatic neuroendocrine neoplasms - management guidelines (recommended by the Polish Network of Neuroendocrine Tumours). Endokrynol Pol. 2013; 64(6): 459-479, doi: 10.5603/EP.2013.0031, indexed in Pubmed: 24431118.

3. Barthet M, Giovannini M, Lesavre N, et al. Endoscopic ultrasound-guided radiofrequency ablation for pancreatic neuroendocrine tumors and pancreatic cystic neoplasms: a prospective multicenter study. Endoscopy. 2019; 51(9): 836-842, doi: 10.1055/a-0824-7067, indexed in Pubmed: 30669161.

4. Mele C, Brunani A, Damascelli B, et al. Non-surgical ablative therapies for inoperable benign insulinoma. J Endocrinol Invest. 2018; 41(2): 153-162, doi: 10.1007/s40618-017-0738-3, indexed in Pubmed: 28755102.

5. Dabkowski K, Gajewska P, Walter K, et al. Successful EUS-guided ethanol ablation of insulinoma, four-year follow-up. Case report and literature review. Endokrynol Pol. 2017; 68(4): 472-479, doi: 10.5603/ep.2017.0053, indexed in Pubmed: 28819950. 